\title{
A Central Limit Theorem for the Number of Degree- $k$ Vertices in Random Maps
}

\author{
Michael Drmota* $\quad$ Konstantinos Panagiotou ${ }^{\dagger}$
}

October 14, 2011

\begin{abstract}
We prove that the number of vertices of given degree in random planar maps satisfies a central limit theorem with mean and variance that are asymptotically linear in the number of edges. The proof relies on an analytic version of the quadratic method and singularity analysis of multivariate generating functions.
\end{abstract}

\section{Introduction}

In this paper we study planar maps, which are connected planar graphs, possibly with loops and multiple edges, together with an embedding in the plane. A map is rooted if a vertex $v$ and an edge $e$ incident with $v$ are distinguished, and are called the root-vertex and root-edge, respectively. The face to the right of $e$ is called the root-face and is usually taken as the outer (or infinite) face. In this paper we only consider rooted maps.

The enumeration of rooted maps is a classical subject, initiated by Tutte in the 1960's, see [15]. In particular he computed the number $M_{n}$ of rooted maps with $n$ edges, proving the formula

$$
M_{n}=\frac{2(2 n) !}{(n+2) ! n !} 3^{n}
$$

Our main interest is the degree distribution of planar maps. Let $d_{n, k}$ be the probability that the root-vertex has degree $k$ in a map with $n$ edges. Then it is known that the limit $d_{k}=\lim _{n \rightarrow \infty} d_{n, k}$ exists. Actually, the values $d_{k}$ are almost explicit. They are given by the generating function

$$
\sum_{k \geq 2} d_{k} u^{k}=\frac{u \sqrt{3}}{\sqrt{(2+u)(6-5 u)^{3}}} .
$$

For more details see Liskovets [13].

The problem is slightly different when we look at the degree of of random vertex in a random map (with $n$ edges). Let $p_{n, k}$ denote the probability that a randomly chosen vertex in a random map with $n$ edges has degree $k$. Then the limit $p_{k}=\lim _{n \rightarrow \infty} p_{n, k}$ exists, too, and we have

$$
p_{k}=\frac{\mu}{k} d_{k}
$$

\footnotetext{
${ }^{*}$ Institute of Discrete Mathematics and Geometry, TU Wien, Wiedner Hauptstr. 8-10, A-1040 Wien, Austria. This author is supported by the Austrian Science Foundation FWF, Project S9604

${ }^{\dagger}$ Max-Planck-Institut für Informatik, Department 1: Algorithms and Complexity, Campus E1.4, 66123 Saarbrücken, Germany.
} 
for a certain constant $\mu>0$. By integration it is possible to obtain an explicit (but involved) representation of the generating function for the sequence $p_{k}$.

Note that $p_{n, k}$ (and consequently $p_{k}$ ) is closely related to the average behavior of the numbers $X_{n}^{(k)}$ of vertices of degree $k$ in a random planar map with $n$ edges. We have $\mathbb{E} X_{n}^{(k)}=$ $p_{n, k} n$ and, thus, as $n \rightarrow \infty$

$$
\mathbb{E} X_{n}^{(k)} \sim p_{k} n
$$

\section{Results}

The main goal of this paper is to study the random variable $X_{n}^{(k)}$ in more detail. In particular we will prove a central limit theorem and tail estimates.

Theorem 2.1. Let $k \in \mathbb{N}$. The number $X_{n}^{(k)}$ of vertices of degree $k$ in a random planar map with $n$ edges satisfies a central limit law, i.e.,

$$
\frac{X_{n}^{(k)}-\mathbb{E}\left[X_{n}^{(k)}\right]}{\left(\operatorname{Var}\left[X_{n}^{(k)}\right]\right)^{1 / 2}} \rightarrow N(0,1),
$$

where $\mathbb{E}\left[X_{n}^{(k)}\right]=\mu_{k} n+O(1)$ and $\operatorname{Var}\left[X_{n}^{(k)}\right]=\sigma_{k}^{2} n+O(1)$, and $\mu_{k}, \sigma_{k}>0$ are computable constants. Moreover, $X_{n}^{(k)}$ has exponential tails, i.e., there is an $\varepsilon_{0}>0$ and a $c_{k}>0$ such that for any $0<\varepsilon<\varepsilon_{0}$

$$
\operatorname{Pr}\left[\left|X_{n}^{(k)}-\mathbb{E}\left[X_{n}^{(k)}\right]\right| \geq \varepsilon \mathbb{E}\left[X_{n}^{(k)}\right]\right] \leq e^{-\varepsilon^{2} c_{k} n} .
$$

Since the dual of a planar map is again a planar map and the degree of a vertex corresponds to the valency of a face in the dual the same result holds for the number of faces of given valency. Actually our combinatorial approach will make use of this correspondence.

Tail estimates for $X_{n}^{(k)}$ have been also obtained by Gao and Wormald [11]. They are on the one hand weaker but on the other hand uniform for $k \leq c \log n$ (for a proper constant $c>0$ ). However, the central limit theorem was unknown.

Nevertheless, there are several classes of planar maps and graphs, where a central limit theorem holds. For example, Gao and Wormald [12] showed such a result for certain classes of triangulations. Moreover, for labelled outerplanar graphs and labelled series-parallel graphs this was shown by Drmota, Gimenéz and Noy [6]. This result was extended to so-called subcritical graph classes by Drmota, Fusy, Kang, Kraus and Rue [5], even in the unlabelled case.

For random planar graphs the current picture is unfortunately incomplete. It was shown by Drmota, Gimenez and Noy [7] and Panagiotou and Steger [14] that the limiting degree distribution exists (and that there is at least a weak concentration result for $X_{n}^{(k)}[14]$ ) but at the moment - there is no central limit theorem although there is no doubt that a central limit theorem should hold. Indeed, there is a strong relation between planar maps and planar graphs. For example, by Whitney's theorem 3-connected planar maps and 3-connected planar graphs coincide. It is therefore very likely that several shape characteristics have (up to a scaling constant) the same limiting behaviour. Hence our results supports the conjecture that there is also a central limit theorem for planar graphs. 
Plan of the paper The paper is structured as follows. In the next section we present the basic equations that are satisfied by the ordinary generating function enumerating planar maps, where also the number of faces of a certain valency. Then, in Section 4 we present the quadratic method and an extension thereof, which is is necessary for our purposes. Finally, in the main result (Theorem 2.1) is shown in Section 5.

\section{Combinatorics}

We use ordinary generating functions, where $z$ marks edges and $x$ non-root faces. The next statement is classical in the area of map enumeration (and goes back to Tutte [15]). We include a proof for completeness, as we shall use similar considerations in Lemma 3.2 to determine a functional equation for the generating function that takes faces of degree $k$ into account, too. Note that it is sufficient to study the valency distribution of faces, it is the same as the degree distribution.

Lemma 3.1. Let $M(z, x, u)$ be the ordinary generating function enumerating general maps with respect to edges and non-root faces, where additionally $u$ marks the degree of the root face. Then

$$
M(z, x, u)=1+z u^{2} M(z, x, u)^{2}+\frac{z x u}{1-u}(M(z, x, 1)-u M(z, x, u)) .
$$

Proof. We replicate the argument by Tutte [15], tailored to our specific purpose. A general map belongs to precisely one of the following three categories. First, it contains no edge, so that the corresponding generating function is the constant 1. Second, the root edge is a bridge, i.e., if removed, the map falls apart in two general maps. Clearly, the generating function enumerating such maps is given by $z u^{2} M(z, x, u)^{2}$.

All maps that belong to none of the two categories above are obtained by taking a map and adding an edge that preserves its root node and "cuts across" the root face in some unambiguous fashion, i.e., so that the construction can be reverted. This operation results in $r+1$ new distinct maps with root-face degrees in $\{1, \ldots, r+1\}$, and one edge and one non-root face more than the map we started with; see Figure 1 for an illustration.

By putting everything together we infer that this construction translates the monomial $u^{r}$ to

$$
z x\left(u+u^{2}+\cdots+u^{r+1}\right)=\frac{z x u\left(1-u^{r+1}\right)}{1-u} .
$$

Consequently, the maps in the last category are enumerated by $\frac{z x u}{1-u}(M(z, x, 1)-u M(z, x, u))$. This completes the proof.

In a second step we also take into account the number of (non-root) faces of valency $k$. A similar, though not so explicit, result was shown by Bender and Canfield [1] and BousquetMélou and Jehanne [2].

Lemma 3.2. Let $k \geq 2$ be a fixed integer and let $M(z, x, w, u)$ be the ordinary generating function enumerating general maps with respect to edges and non-root faces, where additionally $u$ marks the degree of the root face and $w$ the non-root faces of valency $k$. Then

$$
\begin{aligned}
M(z, x, w, u) & =1+z u^{2} M(z, x, w, u)+\frac{z x u}{1-u}(M(z, x, w, 1)-u M(z, x, w, u)) \\
& +z x(w-1) u^{k} M(z, x, w, 1)-z x(w-1) u^{k}\left[q^{k}\right] \frac{q^{2}}{1-q} G(q),
\end{aligned}
$$




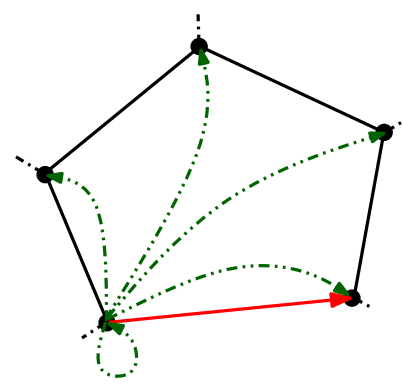

Figure 1: All maps that can be obtained by "cutting across" the root face of size five.

where $G(q)$ is given by the quadratic equation

$$
G(q)=1+z q^{2} G(q)^{2}+z x M(z, x, w, 1) \frac{q}{1-q}-z x \frac{q^{2}}{1-q} G(q) .
$$

Proof. For the sake of brevity let us write $M(u)$ for $M(x, z, w, u)$ and $M_{\ell}=M_{\ell}(x, z, w)$ for the coefficient $\left[u^{\ell}\right] M(u)$. By using the same decomposition as in the proof of Lemma 3.1 we get

$$
M(u)=1+z u^{2} M(u)^{2}+\frac{z x u}{1-u}(M(1)-u M(u))+z x(w-1) u^{k}\left(M(1)-\sum_{\ell=0}^{k-2} M_{\ell}\right) .
$$

The difference to the proof of Lemma 3.1 is that $u^{r}$ is replaced now by

$$
z x\left(u+u^{2}+\cdots+u^{r+1}\right)+z x(w-1) u^{k}=\frac{z x u\left(1-u^{r+1}\right)}{1-u}+z x(w-1) u^{k}
$$

if $r \geq k-1$ (and by $z x\left(u+u^{2}+\cdots+u^{r+1}\right)$ if $r<k-1$ ).

What we show next is that $M_{\ell}=M_{\ell}(z, x, w)$ can be computed easily for $\ell \leq k-2$. Of course we have $M_{0}=1$ and by differentiating (3.3) with respect to $u$ and by setting $u=0$ we get $M_{1}=z x M(1)$. Furthermore, for $2 \leq \ell \leq k-2$ we obtain the recurrence

$$
M_{\ell}=z \sum_{j=0}^{l-2} M_{j} M_{\ell-j}+z x M(1)-z x \sum_{j=0}^{\ell-2} M_{j}
$$

Hence, we can compute $M_{\ell}$ recursively and observe also that $M_{\ell}$ can be written as a polynomial in $z, x$, and $M(1)$.

Actually we can be more precise. By extending (3.4) formally to all $\ell \geq 2$ and by introducing (formally) the generating function $G(q)=\sum_{\ell \geq 0} M_{\ell} q^{\ell}$ we obtain (3.2).

\section{Analytic Quadratic Method}

We first recall the principle of the quadradic method (that is due to Brown [3]). Suppose that we fix $x=x_{0}=1$ and $w=w_{0}=1$ in the equation (3.1). Furthermore we use the abbreviations 
$M(z, u)=M\left(z, x_{0}, w_{0}, u\right)$ and $y(z)=M\left(z, x_{0}, w_{0}, 1\right)$. By completing the square the equation (3.1) can be rewritten as

$$
\left[G_{1}(z, u) M(z, u)+G_{2}(z, u)\right]^{2}=H(z, y(z), u)
$$

where the $G_{i}$ and $H$ are polynomials (more generally it is sufficient to assume that $G_{i}$ and $H$ are analytic function and it is also possible to assume that the $G_{i}$ depend on $y(z)$, too). For example, if $x=x_{0}=1$ and $w=w_{0}=1$ then

$$
H=4(u-1) u^{3} z^{2} y+u^{4} z^{2}-4 u^{4} z+6 u^{3} z-2 u^{2} z+u^{2}-2 u+1 .
$$

The quadratic method consists on binding variables $z$ and $u$, assuming that there exists a function $u(z)$ such that $H(z, y(z), u(z))=0$ identically. Because of the square in the left-hand side of (4.1), the derivative $H_{u}(z, y(z), u(z))$ with respect to $u$ also vanishes. From the system of equations

$$
H(z, y(z), u(z))=0, \quad H_{u}(z, y(z), u(z))=0 .
$$

one eliminates $y(z)$ to find $u(z)$, and then find $y(z)$ from $H(z, y(z), u(z))=0$. Once we know $y(z)=M(z, 1)$, from equation (3.1) we obtain $M(z, u)$.

If we carry out this program in this particular case of maps (recall that we set $x=x_{0}=1$ and $\left.w=w_{0}=1\right)$, we find that $u(z)=(5-\sqrt{1-12 z}) /(2(z+2))$ and

$$
y(z)=\frac{18 z-1+(1-12 z)^{3 / 2}}{54 z^{2}}=1+2 z+9 z^{2}+54 z^{3}+\cdots,
$$

from which we can deduce the explicit form for the numbers $M_{n}$. An explicit expression is obtained also for $M(z, u)$, which encodes completely the distribution of the degree of the root-face. Since planar maps are closed under duality, this is the same distribution as the degree of the root-vertex. It is an easy exercise to derive the limiting distribution encoded in the generating function (1.1).

The system (4.3) can be also used to detect the singularity $z_{0}=1 / 12$ of $y(z)$. We have to look at critical points $\left(z_{0}, y_{0}, u_{0}\right)$ of the system (4.3). Its Jacobian

$$
\left|\begin{array}{cc}
H_{y} & H_{u} \\
H_{u y} & H_{u u}
\end{array}\right|=\left|\begin{array}{cc}
H_{y} & 0 \\
H_{u y} & H_{u u}
\end{array}\right|=H_{y} H_{u u}
$$

must vanish, that is, $H_{y} H_{u u}=0$ at $\left(z_{0}, y_{0}, u_{0}\right)$. It turns out that $H_{u u}=0$ and $H_{y} \neq 0$ is the correct choice for map counting problems. The critical condition is then

$$
H_{u u}\left(z_{0}, y_{0}, u_{0}\right)=0 .
$$

This condition is easy to check, since we always work in the realm of algebraic functions and algebraic numbers. Actually the system $H=H_{u}=H_{u u}=0$ has (usually) only finitely many solutions. For the running example we are using, we have $\left(z_{0}, y_{0}, u_{0}\right)=(1 / 12,6 / 5,4 / 3)$ and A simple check gives $H_{u u}(1 / 12,6 / 5,4 / 3)=0$.

The most interesting observation in this context is that $y(z)$ has a singularity of the kind $\left(1-z / z_{0}\right)^{3 / 2}$. This behavior is typical in the context of planar map enumeration, and it turns out that it there is also a universal analytic reason for this behavior. Recently this was observed by Drmota and Noy [8]. They proved the following lemma. For the reader's convenience we include a proof, since we have to generalize it. ${ }^{1}$

\footnotetext{
${ }^{1}$ The proof is not included in the final version of the proceedings paper [8].
} 
Lemma 4.1. Suppose that $y_{0}, z_{0}, u_{0}$ are complex numbers and that $H(z, y, u)$ is a function that is analytic at $\left(z_{0}, y_{0}, u_{0}\right)$ and satisfies the properties

$$
H\left(y_{0}, z_{0}, u_{0}\right)=0, \quad H_{u}\left(y_{0}, z_{0}, u_{0}\right)=0, \quad H_{u u}\left(y_{0}, z_{0}, u_{0}\right)=0
$$

and

$$
H_{y} \neq 0, \quad H_{u y} \neq 0, \quad H_{u u u} \neq 0, \quad H_{z} H_{u y} \neq H_{y} H_{u z}
$$

for $(y, z, u)=\left(y_{0}, z_{0}, u_{0}\right)$. Then the system of functional equations

$$
H(y(z), z, u(z))=0, \quad H_{u}(y(z), z, u(z))=0
$$

has precisely two (local) solutions $u(z)$ and $y(z)$ with $u\left(z_{0}\right)=u_{0}$ and $y\left(z_{0}\right)=y_{0}$ which are given by

$$
u(z)=g_{1}(z) \pm g_{2}(z) \sqrt{1-\frac{z}{z_{0}}}, \quad y(z)=h_{1}(z) \pm h_{2}(z)\left(1-\frac{z}{z_{0}}\right)^{3 / 2}
$$

in a neighbourhood of $z_{0}$ (except in the part, where $1-z / z_{0} \in \mathbb{R}^{-}$), where $g_{1}(z), g_{2}(z), h_{1}(z)$, and $h_{2}(z)$ are analytic functions at $z_{0}$ and satisfy

$$
\begin{aligned}
& g_{1}\left(z_{0}\right)=u_{0}, \\
& g_{2}\left(z_{0}\right)=\sqrt{\frac{2 z_{0}\left(H_{y} H_{u z}-H_{z} H_{u y}\right)}{H_{y} H_{u u u}}} \neq 0, \\
& h_{1}\left(z_{0}\right)=y_{0}, \\
& h_{2}\left(z_{0}\right)=g_{2}\left(z_{0}\right) \frac{2 z_{0}\left(H_{y} H_{u z}-H_{z} H_{u y}\right)\left(H_{u u u} H_{u y}^{2}-H_{y} H_{u y} H_{u u u u}+3 H_{y} H_{u u y} H_{\text {uuu }}\right)}{3 H_{u u u} H_{u y}^{2} H_{y}^{2}}
\end{aligned}
$$

(and all derivatives of $H$ have to be evaluated at $(y, z, u)=\left(y_{0}, z_{0}, u_{0}\right)$, respectively).

Proof. We solve the system (4.4) by first considering the equation $H_{u}(y, z, u)=0$, where $z$ and $u$ are considered as independent variables and $y=Y(z, u)$ is the unknown function. In a second step we solve the equation $H(Y(z, u), z, u)=0$, where $z$ is the independent variable. Then the solution $u=u(z)$ is the function that we are looking for and $y(z)=Y(z, u(z))$.

Since we assume that $H_{u y}\left(y_{0}, z_{0}, u_{0}\right) \neq 0$ it follows from the implicit function theorem that there exists an function $Y(z, u)$ with $Y\left(z_{0}, u_{0}\right)=y_{0}$ that is analytic at $\left(y_{0}, u_{0}\right)$ and solves (locally) the equation $H_{u}(Y(z, u), z, u)=0$. Observe that

$$
\begin{aligned}
& Y_{u}\left(z_{0}, u_{0}\right)=-\frac{H_{u u}}{H_{u y}}=0, \quad Y_{u u}\left(z_{0}, u_{0}\right)=-\frac{H_{u u u}}{H_{u y}} \neq 0, \quad Y_{u u}\left(z_{0}, u_{0}\right)=\frac{3 H_{u u y} H_{u u u}-H_{u y} H_{u u u u}}{H_{u y}^{2}}, \\
& Y_{z}\left(z_{0}, u_{0}\right)=-\frac{H_{u z}}{H_{u y}}, \quad Y_{u z}\left(z_{0}, u_{0}\right)=\frac{H_{u u y} H_{u z}-H_{u u z} H_{u y}}{H_{u y}^{2}}
\end{aligned}
$$

evaluated at $(y, z, u)=\left(y_{0}, z_{0}, u_{0}\right)$. Next we set $F(z, u)=H(Y(z, u), z, u)$ and solve the equation $F(z, u)=0$ for $u=u(z)$. By assumption we have

$$
\begin{aligned}
F\left(z_{0}, u_{0}\right) & =H\left(y_{0}, z_{0}, u_{0}\right)=0, \\
F_{u}\left(z_{0}, u_{0}\right) & =H_{y}\left(y_{0}, z_{0}, u_{0}\right) y_{u}\left(z_{0}, u_{0}\right)+H_{u}\left(y_{0}, z_{0}, u_{0}\right)=0, \\
F_{u u}\left(z_{0}, u_{0}\right) & =H_{y}\left(y_{0}, z_{0}, u_{0}\right) Y_{u u}\left(z_{0}, u_{0}\right) \neq 0, \\
F_{z}\left(z_{0}, u_{0}\right) & =H_{y}\left(y_{0}, z_{0}, u_{0}\right) Y_{z}\left(z_{0}, u_{0}\right)+H_{z}\left(y_{0}, z_{0}, u_{0}\right)=\frac{\left(H_{z} H_{u y}-H_{y} H_{u z}\right)\left(y_{0}, z_{0}, u_{0}\right)}{H_{u y}\left(y_{0}, z_{0}, u_{0}\right)} \neq 0 .
\end{aligned}
$$


Hence the equation $F(z, u)=0$ satisfies the assumptions of a classical lemma on the singular structure of the solution of a single equation (for example, it can be found in [4]). Thus, the only two solutions $u(z)$ have local expansions of the form

$$
u(z)=g_{1}(z) \pm g_{2}(z) \sqrt{1-\frac{z}{z_{0}}},
$$

where $g_{1}(z)$ and $g_{2}(z)$ are analytic and satisfy $g_{1}\left(z_{0}\right)=u_{0}$ and

$$
g_{2}\left(z_{0}\right)=\sqrt{\frac{2 z_{0} F_{z}\left(u_{0}, z_{0}\right)}{F_{u u}\left(u_{0}, z_{0}\right)}}=\sqrt{\frac{2 z_{0}\left(H_{y} H_{u z}-H_{z} H_{u y}\right)}{H_{y} H_{u u u}}} .
$$

A simple calculation (by using Taylor's theorem and by comparing coefficients) we also obtain an expression for

$$
\begin{aligned}
g_{1}^{\prime}\left(z_{0}\right)=\frac{F_{z} F_{u u u}-}{F_{u u}^{2}} & 3 F_{u z} F_{u u} \\
=\frac{1}{3 H_{y}^{2} H_{u u u}^{2}}( & -3 H_{y}^{2} H_{u u u} H_{u u z}+2 H_{u u u} H_{u y} H_{y} H_{u z}-2 H_{u u u} H_{u y}^{2} H_{z} \\
& \left.\quad+3 H_{y} H_{\text {uuy }} H_{u u u} H_{z}+H_{y}^{2} H_{u u u u} H_{u z}-H_{y} H_{u y} H_{u u u u} H_{z}\right) .
\end{aligned}
$$

Finally we use the expansion of $u(z)$ to derive the local behaviour of

$$
\begin{aligned}
y(z) & =Y(z, u(z)) \\
& =y_{0}+Y_{z}\left(z_{0}, u_{0}\right)\left(z-z_{0}\right)+\frac{1}{2} Y_{u u}\left(z_{0}, u_{0}\right)\left(u(z)-u_{0}\right)^{2} \\
& +Y_{u z}\left(z_{0}, u_{0}\right)\left(z-z_{0}\right)\left(u(z)-u_{0}\right)+\frac{1}{6} Y_{\text {uиu }}\left(z_{0}, u_{0}\right)\left(u(z)-u_{0}\right)^{3}+O\left(\left(z-z_{0}\right)^{2}\right) .
\end{aligned}
$$

Note that the property $Y_{u}\left(z_{0}, u_{0}\right)=0$ implies that $y(z)$ has no $\sqrt{1-z / z_{0}}$ term in its expansion. Precise expressions for the coefficients (like $h_{2}\left(z_{0}\right)$ ) can be determined easily.

For completeness, we check all the condition in the statement for $H(z, y, u)$ as in (4.2), evaluated at the critical point $\left(z_{0}, y_{0}, u_{0}\right)=(1 / 12,4 / 3,6 / 5)$. In addition to $H$, we need $G_{1}=2(1-u) u^{2} z$. Then

$$
\begin{gathered}
G_{1}=-\frac{6}{125}, \quad H_{y}=\frac{6}{625}, \quad H_{u y}=\frac{9}{125}, \quad H_{\text {uиu }}=-\frac{50}{9}, \quad H_{z} H_{u y}-H_{y} H_{z u}=\frac{288}{15625}, \\
H_{\text {uuи }} H_{u y}^{2}-H_{y} H_{u y} H_{\text {uuu }}+3 H_{y} H_{\text {uuy }} H_{\text {uuu }}=\frac{43}{625} .
\end{gathered}
$$

Hence we can apply Lemma 4.1 to obtain the singular behaviour of $y(z)=M(z, 1)$ which is of the form $\left(1-z / z_{0}\right)^{3 / 2}$.

In general it is not immmediately clear which critical point $\left(z_{0}, y_{0}, u_{0}\right)$ is responsible for the dominating singularity on the radius of convergence of $y(z)=M(z, 1)$ (when there are several ones). Nevertheless in the case of planar maps there is no doubt that we have chosen the correct critical point. Moreover, we can vary the variables $x$ and $w$ (at least) in a (small) neighbourhood of $x_{0}=1$ and $w_{0}=1$ without changing the kind of the dominating singularity. For this purpose we rewrite (3.1) in the form

$$
\left(G_{1}(z, u) M(z, x, w, u)+G_{2}(z, u)\right)^{2}=\bar{H}(z, x, w, \bar{y}(z, x, w), u),
$$


where $\bar{y}(z, x, w)$ abbreviates $M(z, x, w, 1)$. As above we will consider the system of equations

$$
\bar{H}=\bar{H}_{u}=\bar{H}_{u u}=0,
$$

and consider (if possible the solutions) $y_{0}(x, w), z_{0}(x, w), u_{0}(x, w)$.

Lemma 4.2. There exist complex neighbourhoods $X$ and $W$ of $x_{0}=1$ and $w_{0}=1$ such that the system of equations (4.6) has a unique solution $y_{0}(x, w), z_{0}(x, w), u_{0}(x, w)$ with $y_{0}\left(x_{0}, w_{0}\right)=y_{0}, z_{0}\left(x_{0}, w_{0}\right)=z_{0}, u_{0}\left(x_{0}, w_{0}\right)=u_{0}$. Furthermore, the function $\bar{y}(z, x, w)=$ $M(z, x, w, 1)$ has a local representation of the form

$$
\bar{y}(z, x, w)=\bar{h}_{1}(z, x, w)+\bar{h}_{2}(z, x, w)\left(1-\frac{z}{z_{0}(x, w)}\right)^{3 / 2}
$$

where $\bar{h}_{1}(z, x, w)$ and $\bar{h}_{2}(z, x, w)$ are non-zero analytic functions, and there exists an analytic continuation to

$$
\Delta=\left\{z:|z|<\left|z_{0}(x, w)\right|+\eta,\left|\arg \left(z / z_{0}(x, w)-1\right)\right|>\delta\right\},
$$

for some real numbers $\eta>0$ and $0<\delta<\pi / 2$.

Proof. The first step is to show that the system of equation (4.6) has a proper solution in a neighbourhood of $x_{0}=1$ and $w_{0}=1$. Actually we know that it has a solution for $x=1$ and $w=1$. Furthermore the Jacobian

$$
\left|\begin{array}{ccc}
\bar{H}_{y} & \bar{H}_{z} & \bar{H}_{u} \\
\bar{H}_{u y} & \bar{H}_{u z} & \bar{H}_{u u} \\
\bar{H}_{u u y} & \bar{H}_{\text {uuz }} & \bar{H}_{\text {uuu }}
\end{array}\right|=\left|\begin{array}{ccc}
\bar{H}_{y} & \bar{H}_{z} & 0 \\
\bar{H}_{u y} & \bar{H}_{u z} & 0 \\
\bar{H}_{u u y} & \bar{H}_{u u z} & \bar{H}_{\text {uuu }}
\end{array}\right|=\bar{H}_{\text {uuu }}\left(\bar{H}_{y} \bar{H}_{u z}-\bar{H}_{z} \bar{H}_{u y}\right) \neq 0
$$

for $x=x_{0}=1$ and $w=w_{0}=1$. Hence, by the implicit function theorem there exists a neighbourhood $X$ of $x_{0}=1$ and neighbourhood $W$ of $w_{0}=1$ such that the system (4.6) has an analytic solution $y_{0}(x, w), z_{0}(x, w), u_{0}(x, w)$ with $y_{0}\left(x_{0}, w_{0}\right)=y_{0}, z_{0}\left(x_{0}, w_{0}\right)=z_{0}$, $u_{0}\left(x_{0}, w_{0}\right)=u_{0}$. By continuity we can choose $X$ and $W$ also in a way that the non-zero conditions $\left(\bar{H}_{y} \neq 0\right.$ etc. $)$ of Lemma 4.1 are satisfied in $X$ and $W$.

By checking the proof of Lemma 4.1 it follows that it generalizes to $x \in X$ and $w \in W$ so that all appearing functions are analytic in $x$ and $w$. In particular we use that fact that this is true for equations of the form $F(z, x, w, u)=0$, where we observe that the solution $u=u(z, x, w)$ can be locally represented as

$$
u(z, x, w)=g_{1}(z, x, w)-g_{2}(z, x, w) \sqrt{1-\frac{z}{z(x, w)}}
$$

with analytic functions $g_{1}(z, x, w)$ and $g_{2}(z, x, w)$ (for details see [4]). This leads to (4.7).

Finally we have to check that $\bar{y}(z, x, w)$ has a proper analytic continatation to the region $\Delta$. For this purpose we have to study $y(z)$ not only around $z=z_{0}$. Since we know $y(z)$ and $u(z)$ explicitly it is easy to check that $H_{u u}(z, y(z), u(z)) \neq 0$ if $z \neq z_{0}$ and $H_{y}(z, y(z), u(z)) \neq 0$ if $z \neq z_{0}$. Hence the Jacobian

$$
\left|\begin{array}{cc}
H_{y} & H_{u} \\
H_{u y} & H_{u u}
\end{array}\right|=\left|\begin{array}{cc}
H_{y} & 0 \\
H_{u y} & H_{u u}
\end{array}\right|=H_{y} H_{u u}
$$


is non-zero for $|z|=z_{0}$ but $z \neq z_{0}$.

Now suppose that $x$ and $w$ vary in properly chosen neighbourhoods of $x_{0}=1$ and $w_{0}=1$. If $\left|z-z_{0}(x, w)\right|<\varepsilon$ we use the local representation (4.7) and obtain an analytic continuation. If $|z|$ is close to $\left|z_{0}(x, w)\right|$ but $\left|z-z_{0}(x, w)\right| \geq \varepsilon$ we obtain by continuity that the Jacobian $\bar{H}_{u u} \bar{H}_{y}$ stays non-zero (if $x$ and $w$ are sufficiently close to $x_{0}=1$ and $w_{0}=1$ ). Hence, by the implicit function theorem $y(z, x, w)$ and $u(z, x, w)$ can be analytically continued. By compactness it is sufficient to continue $y(z, x, w)$ (and $u(z, x, w)$ only at finitely many points. Hence, there exist $\delta>0$ and $\eta>0$ such that $y(z, x, w)$ continues analytically to $\Delta$.

\section{The Central Limit Theorem}

It is now easy to complete the proof of Theorem 2.1. For this purpose we use the following transfer principle by Flajolet and Odlyzko [9] (see also the book of Flajolet and Sedgewick [10] and many references therein).

Lemma 5.1. Let $W$ be a compact set and assume that there exist functions $C(w), z_{0}(w)$, $\alpha(w), \beta(w)$ such that $\beta(w)>\mathfrak{R}(\alpha(w)), \alpha(w) \in \mathbb{C} \backslash \mathbb{N}_{0}$ and $z_{0}(w)>0$ for all $w \in W$ with the following property. Suppose that $f(z ; w)$ is a power series in $z$ and a parameter $w \in W$ such that there is an expansion of the form

$$
f(z ; w)=C(w)\left(1-\frac{z}{z_{0}(w)}\right)^{\alpha(w)}+O\left(\left(1-\frac{z}{z_{0}(w)}\right)^{\beta(w)}\right)
$$

that is uniform for $w \in W$ and $z \in \Delta$, where

$$
\Delta=\left\{z:|z|<\left|z_{0}(w)\right|+\eta,\left|\arg \left(z / z_{0}(w)-1\right)\right|>\delta\right\},
$$

for some real numbers $\eta>0$ and $0<\delta<\pi / 2$. Then, uniformly for $w \in W$

$$
\left[z^{n}\right] f(z ; w)=(1+o(1)) \cdot C(w) \frac{n^{-\alpha(w)-1}}{\Gamma(-\alpha(w))} z_{0}(w)^{-n} .
$$

With the help of this lemma we can prove the following property.

Lemma 5.2. Let $k \geq 2$ and $X_{n}^{(k)}$ as in Theorem 2.1. Then there exists a neighbourhood $W$ of $w_{0}=1$ such that

$$
\mathbb{E}\left(w^{X_{n}^{(k)}}\right)=(1+o(1)) C(w)\left(\frac{z_{0}}{z_{0}\left(x_{0}, w\right)}\right)^{n}
$$

uniformly for $w \in W$, where $C(w)$ is a non-zero analytic function.

Proof. We apply this Lemma 5.1 for $f(z ; w)=\bar{y}\left(z, x_{0}, w\right)-\bar{h}_{1}\left(z, x_{0}, w\right)$, which can be rewritten as

$$
f(z ; w)=\bar{h}_{2}\left(z_{0}\left(x_{0}, w\right), x_{0}, w\right)\left(1-\frac{z}{z_{0}\left(x_{0}, w\right)}\right)^{3 / 2}+O\left(\left(1-\frac{z}{z_{0}\left(x_{0}, w\right)}\right)^{5 / 2}\right) .
$$

The additive term $\bar{h}_{1}\left(z, x_{0}, w\right)$ is analytic in a larger region and, thus, provides a negligible contribution. Consequently we obtain

$$
\left[z^{n}\right] \bar{y}\left(z, x_{0}, w\right)=(1+o(1)) \bar{h}_{2}\left(z_{0}\left(x_{0}, w\right), x_{0}, w\right) \frac{n^{-5 / 2}}{\Gamma(-3 / 2)} z_{0}\left(x_{0}, w\right)^{-n} .
$$


and also

$$
\mathbb{E}\left(w^{X_{n}^{(k)}}\right)=\frac{\left[z^{n}\right] \bar{y}\left(z, x_{0}, w\right)}{\left[z^{n}\right] \bar{y}\left(z_{0}, x_{0}, w_{0}\right)}=(1+o(1)) \frac{\bar{h}_{2}\left(z_{0}\left(x_{0}, w\right), x_{0}, w\right)}{\bar{h}_{2}\left(z_{0}, x_{0}, w_{0}\right)}\left(\frac{z_{0}}{z_{0}\left(x_{0}, w\right)}\right)^{n} .
$$

By Hwang's Quasi-Power-Theorem (see [10] or [4]) the central limit theorem (and also the exponential tail estimates) follow immediately.

\section{References}

[1] E. A. Bender and E. R. Canfield, The Number of Degree-Restricted Rooted Maps on the Sphere, SIAM J. Discrete Math. 7 (1) (1994) 9-15.

[2] M. Bousquet-Mélou and A. Jehanne, Polynomial equations with one catalytic variable, algebraic series and map enumeration, J. Comb. Theory, Ser. B 96 (5) (2006) 623-672.

[3] W.G. Brown, On the existence of square roots in certain rings of power series, Math. Annalen 158 (1965) 82-89.

[4] M. Drmota. Random Trees: an Interplay between Combinatorics and Probability, Springer, 2009.

[5] M. Drmota, E. Fusy, M. Kang, V. Kraus, and J. Rue, Asymptotic study of subcritical graph classes, SIAM J. Dicrete Math., to appear.

[6] M. Drmota, O. Gimenez and M. Noy, Vertices of given degree in series-paralles graphs, Random Structures Algorithms., 36 (2010), 273-314.

[7] M. Drmota, O. Gimenez and M. Noy, Degree distribution in random planar graphs, J. Combinatorial Theory A, 118 (2011), 2102-2130.

[8] M. Drmota and M. Noy, Universal exponents and tail estimates in the enumeration of planar maps, Proceedings of Eurocomb 2011, to appear.

[9] P. Flajolet and A. Odlyzko, Singularity analysis of generating functions, SIAM J. Discrete Math. 3 (1990), 216-240.

[10] F. Flajolet and R. Sedgewick, Analytic Combinatorics, Cambridge University Press, 2009.

[11] Z. Gao and N. Wormald Sharp concentration of the number of submaps in random planar triangulations, Combinatorica, 23 (3) (2003), 467-486.

[12] Z. Gao and N. Wormald Asymptotic normality determined by high moments, and submap counts of random maps, Probab. Theory Related Fields, 130 (3) (2004), 368-376.

[13] V. A. Liskovets, A pattern of asymptotic vertex valency distributions in planar maps, J. Combin. Theory Ser. B 75 (1999), 116-133.

[14] K. Panagiotou and A. Steger On the Degree Distribution of Random Planar Graphs, Proceedings of the 22nd Annual ACM-SIAM Symposium on Discrete Algorithms (SODA '11), (2011), 11981210 .

[15] W. T. Tutte, A census of planar maps, Canad. J. Math. 15 (1963), 249-271. 